\title{
Simulation Model of Bus Rapid Transit
}

\author{
Fergyanto E. Gunawan $^{1, a}$, Suharjito ${ }^{1}$ and Alexander A S Gunawan ${ }^{2}$ \\ ${ }^{1}$ Binus Graduate Programs, Bina Nusantara University, 11480 Jakarta, Indonesia \\ ${ }^{2}$ School of Computer Science, Bina Nusantara University, 11480 Jakarta, Indonesia
}

\begin{abstract}
Bus rapid transit system is modern solution for mass transportation system. The system, in comparison to the rail-based transportation system, is significantly cheaper and requires shorter development time, but lower performance. The BRT system performance strongly depends on variables related to station design and infrastructure. A numerical model offers an effective and efficient means to evaluate the system performance. This article offers a detailed numerical model on the basis of the discrete-event approach and demonstrates its application.
\end{abstract}

\section{Introduction}

The key to solve traffic congestion in a megacity lies on development of a modern mass transportation system [1], either a bus-based system or a rail-based system. Since the end of the last century, the bus-based system, so called bus rapid transit (BRT) system, has been deployed across the globe at a higher rate than that of the rail-based system as shown in Figure 1 [2].

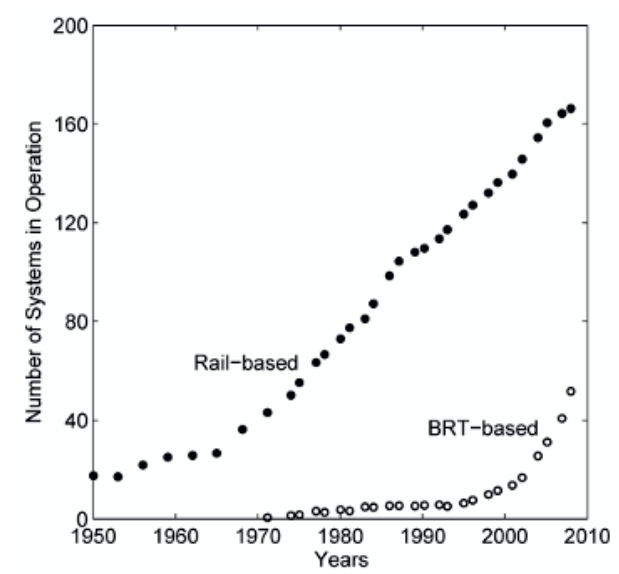

Figure 1. The number of rail-based and bus-based mass transportation systems across the globe [2].

The performance of the BRT system is affected by various factors involving station design, infrastructure, service planning, passenger information systems, and integration and access [3]. For that reason, assessment on the cost-effective measure to improve its performance should be based on accurate and comprehensive evidents, which may be gathered at a reasonable cost by numerical simulation.
Many previous publications had addressed the issue of BRT numerical simulation [4-14]; some deployed a discrete-event approach [7-11], and some deployed a discrete-time approach [12-14]. The discrete-time approach is that of the microsimulation. Based on the earlier approach, ref. [7] evaluated a framework of the intelligent transportation systems, ref. [8] modeled a logistics system in conjunction with virtual reality, ref. [9] optimized a fleet of closed-loop maritime transportation of a steel manufacturing company, ref. [10] modeled a rail-based transportation system, and finally, ref. [11] evaluated different BRT system configurations of new BRT line in Rome.

As for the microsimulation approach, ref. [12] deployed VISSIM-a system based on microsimulation - to model the movement of buses in the Beijing North-South Central Axis corridor, and the model was calibrated by minimizing the relative speed of buses at a number of detector points along the corridor, ref. [13] studied factors influencing public bus travel efficiency in urban traffic in China, and finally, ref. [14] determined a bus limit capacity of a BRT station in Brisbane, Australia using a calibrated AIMSUM microsimulation model.

The present work discusses those existing approaches and presents a detailed development of a DES-based simulation of the BRT system, and demonstrates its application.

The paper is structured as following: Section 2 Fundamental Theory will describe the bus rapid transit system, the discrete-time approach, and also the discreteevent approach for simulation the BRT system. Section 3 Numerical Trials: Simple Corridor demonstrates the application of the developed model using a simple twostation corridor model.

\footnotetext{
a Corresponding author: fgunawan@binus.edu, f.e.gunawan@gmail.com
} 


\section{Fundamental Theory}

\subsection{Bus Rapid Transit}

The bus rapid transit was originated in the city of Curitiba, Brazil in 1974. Since the end of the last century, the system has been receiving a lot interests. For examples: TransMilenio BRT was started in Dec. 2000 in Bogota, TransJakarta BRT was in Jan. 2004 in Jakarta, and Guangzhou BRT in Feb. 2010 [15].

The system is capable to serve large number of passengers within a short amount of time due to various design and operational considerations. Most importantly, the BRT system is designed to have dedicated and physically protected bus lanes in central verge of the road, off-vehicle fare collection, and platform-level boarding [3]. The first should protect the BRT buses from a mixed traffic; therefore, allows them to travel undisturbed along their bus way. The second will cut considerable amount of time for fare transfer existing in the traditional bus system. The third allows the BRT passengers to move quickly between the bus platform and the BRT bus.

\subsection{Discrete-Time Approaches}

The discrete-time traffic modeling may employ a model in microscopic scale, where individual vehicles and their interactions are described by ordinary differential equations, mesoscopic models where vehicle interactions are described by a statistical mechanics, and macroscopic models where the traffic flow is described similar to continuum fluid. However, all of these models are related [16].

\subsubsection{Macroscopic Simulation}

In the macroscopic model, we commonly use the PayneWhitham equation of [17]:

$$
\rho_{t}+(u \rho)_{x}=0
$$

where the subscripts denoting differentiation in space and in time for $x \in[0, L]$ where $L$ is the length of a road segment. In Eq. (1), $\rho$ is the traffic density, and $u$ is the speed of the traffic flow. Furthermore, we also have to assume the fundamental relation between interacting variables $u, \rho$ or the traffic flow, $q$. For an example, ref. [18] assumes

$$
u(\rho)=v_{\max }\left[1-\left(\frac{\rho}{\rho_{\max }}\right)^{2}\right]
$$

where $v_{\max }$ is the maximum speed and $\rho_{\max }$ is the maximum traffic density.

However, the case of BRT system is unique due the existing bus way that limits the number vehicles operating in the lane to a level that solving Eq. (1) becomes unnecessary.

\subsubsection{Microscopic Simulation}

The microscopic models seem to be more suitable approach than the macroscopic models. At the microscopic level, the traffic dynamic is considered at each vehicle level, but the most importantly, the vehicle is assumed to move on a prescribed lane, which agrees with the BRT operation. In addition, the change of lanes may exist on few stations having such facilities, and the movement is clearly prescribed. Therefore, the microscopic model is more appropriate then the macroscopic model to model the BRT system.

For an example of the microscopic models is that of the intelligent driver model [31,32], which can be written as:

$$
a_{n}(t)=a_{\max }\left[1-\left(\frac{v_{n}(t)}{v_{0}}\right)^{\delta}-\left(\frac{s^{*}(t)}{s_{n}(t)}\right)^{2}\right]
$$

where $s_{n}$ is the gap of the $n$-vehicle. The variables $a_{n}(t)$ and $v_{n}(t)$ respectively denote the vehicle acceleration and velocity. The gap is the headway space, or mathematically, is expressed as:

$$
s *(t)=s_{0}+s_{1} \sqrt{\frac{v_{n}(t)}{v_{0}}}+T v_{n}(t)+\frac{v_{n}(t) \Delta v_{n}(t)}{2 \sqrt{a_{\max } a_{\min }}}
$$

In total, the IDM acceleration model has eight parameters, which can be categorized into three groups. A parameter in the first group determines the steady state motion of the vehicle; three parameters in the second group determine the vehicle acceleration/deceleration; and finally, four parameters in the last group determine a non-collision gap with respect to the front vehicle. The member of the first group is the vehicle desired velocity ${ }^{v_{0}}$. Meanwhile, the members of the second group are the vehicle maximum acceleration ${ }^{a_{\max }}$, the minimum deceleration $a_{\text {min }}$, and the acceleration exponent $\delta$. As for the last group, the members are the safe time headway $T$, the jam distances $S_{0}$ and ${ }^{S_{1}}$, and the vehicle length $D$. The non-zero $S_{1}$ is only necessary on rather special conditions such as when the equilibrium flow-density requiring an inflection point [32].

\subsection{Discrete-Event Approach}

\subsubsection{Fundamental Model}

To develop BRT sub-systems, we firstly reduce the size of a regular BRT system to a simple model but having all necessary sub-systems of the actual BRT system. This simplest model, we called the canonical model, is depicted in Fig. 2. 


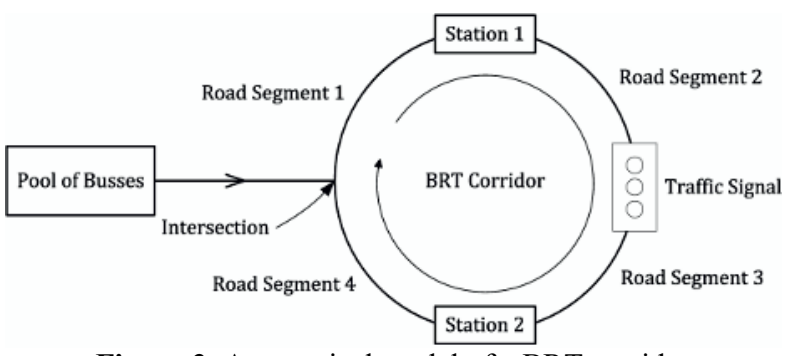

Figure 2. A canonical model of a BRT corridor

The model in Fig. 2 is a simple model of a BRT corridor where each bus that serves the corridor departing from the pool at a scheduled time. The schedule for the modern BRT system should relatively be high in bus frequency particularly during rush-hours (see Table 1). Then, the bus goes to Station 1 via the road-segment 1 . For the case of TransJakarta BRT, the road-segment length varies from 0.3 to $1 \mathrm{~km}$ [19]. The bus stays at Station 1 for some amount of time to drop and pick up the passengers, and then, goes to Station 2. Finally, the bus will circulate in the corridor until it meets the end of the operation time for the day. Modern BRT is also required to operate until late at night [3]. In an actual BRT system, the bus will serve larger number of stations. TransJakarta BRT, for an example, has about 15-26 stations per corridor [19].

Table 1. Modern requirement for bus frequency during peak period. During the time, the BRT Standard awards up to four points for frequency of service in the highest-demand segment [3].

\begin{tabular}{cc}
\hline Service Frequency (minute) & Points \\
\hline$<3$ & 4 \\
$3-5$ & 3 \\
$5-7$ & 2 \\
$7-10$ & 1 \\
\hline
\end{tabular}

It is clear that the canonical model in Fig. 2 has necessary sub-systems to model a corridor of BRT system. Those sub-systems are the station, the roadsegment, the pool of buses, the intersection, and the traffic signal. On the following, we will discuss the development of each BRT sub-system on the basis of Matlab SimEvents blocks.

\subsubsection{The Station Sub-system}

This sub-system represents the BRT station, and it is very critical because this is the place where transit activity mainly occurs. The BRT buses have to stop at the station for the passengers to board and alight, and the station platform should be at same level of the bus platform to reduce the passenger transfer-time.

The BRT system is designed so that the boarding and alighting activities can be performed within a short time. In comparison to the traditional bus system, the time required by the BRT buses is significantly shorter. This is achieved by three important design considerations of BRT system: alignment of the station platform and the bus floor, off-vehicle fare collection, and buses having wide doors [3].
An important feature of this sub-system is that the system should be able to accurately capture the dynamics of passenger arrivals at the station. Fortunately, the issue has been of interest of many researchers, for examples: refs. [20-25]. Some of those literatures had established the dynamics mathematically.

In general, the existing literatures identified the passenger dynamics and established the following conceptions. The times of the passenger arrivals inclined to follow, roughly speaking, two probability distribution functions. They are the uniform and log-normal distribution functions depending on the bus headway.

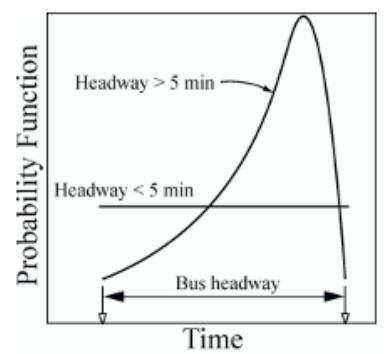

Figure 3. Probability of passenger arrivals as a function of the bus headway

For a short headway, say less than $5 \mathrm{~min}$, the times of arrivals are inclined to follow the uniform distribution function, which means that the passengers arrived randomly. As for a long headway, i.e., longer than $5 \mathrm{~min}$, the times of arrivals are inclined to the log-normal distribution function. In the latter case, the passengers mostly arrived a few minutes before the bus scheduled arrival. Majority of the arrivals occurred about $4 \mathrm{~min}$ before the bus schedule. Many have identified that the 5min-headway time as the transition of the arrival patterns. We should note that ref. [22] identified the headway of 10 minutes as the transition between the two distributions.

Those literatures, some are traditional, established the passengers waiting time $(w)$ as a function of the bus headway $\left({ }^{h}\right)$. Most literatures agreed that $w=h / 2$ for a short headway [22]. This particular model was established on top of three assumptions; they were: (i) the passengers arriving randomly, (ii) the bus arriving regularly, and (iii) the passengers get on their first bus.

In reality, the BRT bus headways may vary considerably [26]. To take this aspect into account, refs. [27,28] and ref. [29] advised:

$$
w=\frac{\mu}{2}\left(1+\frac{s^{2}}{\mu^{2}}\right),
$$

where $w$ is the expected passenger waiting times, $\mu$ is the mean headways between the buses, and $s^{2}$ is the variances of headways between the buses.

In addition to the model of Eq. (5), many have proposed various empirical formulas, for examples: 


$$
\begin{aligned}
& w=1.79+0.14 \mu[20] \\
& w=2.34+0.26 \mu[30] \\
& w=2.00+0.30 \mu[21]
\end{aligned}
$$

To achieve those described features, we designed a sub-system as shown in Fig. 4. Essentially, the sub-block consists of two parts: the lower sub-block and the upper sub-block with two different entity-types. The entity in the lower sub-block represents a passenger; meanwhile, the entity in the upper sub-block represents a bus.

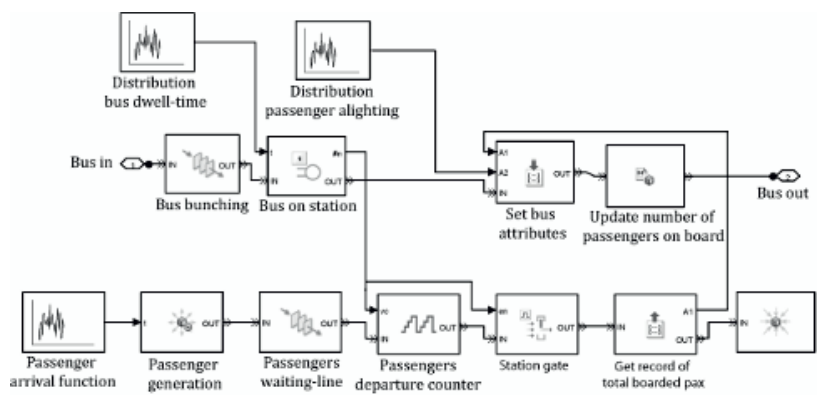

Figure 4. SimEvents block components for the BRT bus station.

The lower sub-block starts with the passenger arrival function. Basically, the function is a statistical function that defines the nature of the passenger arrivals. The block signals the passenger generation block to initiate arrival of passengers. The generated passengers will then be transferred to a FIFO waiting-line where the passengers will wait until the opening of the station gate. The station gate will receive a signal from the upper subblock. The signal will be initiated in the upper sub-block when a bus arrives and dwells at the station.

The upper sub-block starts with a port, which allows a bus to approach the station. The current design of the upper sub-block allows a bus to bunch at the station. The bus bunching is one of the biggest issues faced by the modern BRT system. When the station is empty, the bus will dwell at the station. The dwell-time will be determined by the distribution bus dwell-time block. The bus entity will then receive data regarding the number of alighting passengers and the number of passengers waiting in line to board the bus. Finally, the bus will update those data, which are maintained as the bus attributes data, and departure data from the station to the next station.

\subsubsection{The Road-Segment Sub-system}

This sub-system has to be able to simulate the traveling of a BRT bus along its corridor in a segment of road connecting two adjacent stations. This sub-system is characterized by the bus travel time and the number of buses accommodated by the road segment. Therefore, this sub-system is also modeled with a simple queueserver model having the first-in first-out (FIFO) queuing policy. This approach is appropriate considering the fact that majority of the busway is single lane; hence, a bus has no possibility to overpass other buses.
A number of studies and observations indicate that the travel time for the case can reasonably be approximated with the exponential distribution function with a single controlling parameter of the average travel speed of the bus or the average bus travel time (for an example, see [26]).

Figure 5 shows Matlab SimEvents implementation of the design. The design allows buses to bunch on the roadsegment, and the bus travel time will be determined by a block, which specifies the statistical distribution of the bus travel time.

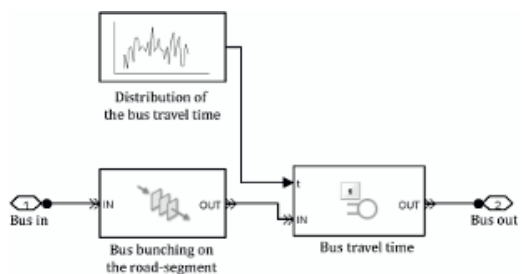

Figure 5. SimEvents block components for the road-segment sub-system.

\subsubsection{The Pool of Buses Sub-system}

This sub-system has to be able to generate entities at a scheduled time where each entity represents a BRT bus. Our current SimEvents implementation is shown in Fig. 6.

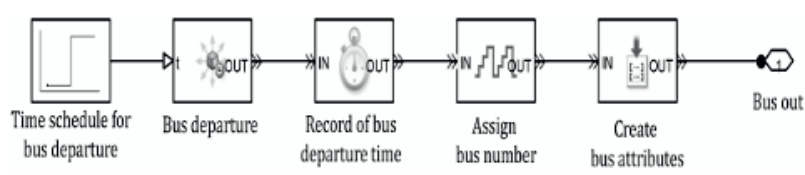

Figure 6. SimEvents block components for the sub-system of the pool of buses.

The sub-system is started with the Time Schedule for Bus Departure Block where the buses departure schedule is specified. The bus schedule specification is deterministic. When the bus schedule has arrived, the Bus Departure Block will release a bus per unit time. Hence, the current design does not allow the bus pool sub-system to serve more than one bus per unit time. Subsequently, the subsystem will record the bus departure time, assign a bus number, and finally, create or attach each bus with some attributes. Those attributes are variables to hold the passenger data. At the current implementation, those attributes are the number of passengers on the bus, and the number of alighted and boarded passengers on the bus last station.

\subsubsection{The Traffic-Signal Sub-system}

This sub-system has to be able to simulate traveling of the BRT buses across a manually controlled traffic-signal. Therefore, the system is characterized by the cycle time of the traffic-signal, $t_{c}$, and the duration of the green signal, $t_{g}$. Also, the sub-system has to allow vehicles queuing for the traffic-signal. 
We designed a sub-system as shown in Fig. 7 to achieve the purpose stated above. Basically, the subsystem consists a FIFO queue block that facilitate the buses to wait for the green light. The FIFO block is then followed by a traffic signal block, which basically is a release gate block. The gate will open according to the time set by the traffic signal timing block. When the gate is open, the bus is allowed to move forward. The timing block is a time function regulated by two variables: the traffic-signal cycle-time and the green-signal duration.

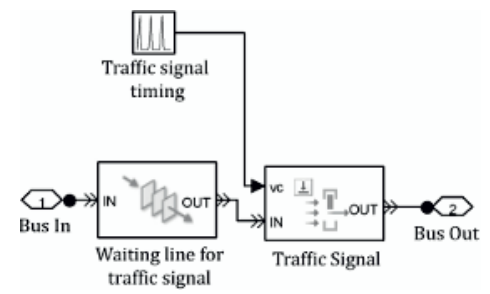

Figure 7. SimEvents block components for the traffic signal sub-system.

Figure 8 demonstrates the use of this sub-system. For this case, 30 vehicles were released from a pool, the cycle time was set to $20 \mathrm{~s}$, and the duration of the green signal was set to $5 \mathrm{~s}$. The figure indicates that the sub-system allowed vehicles to travel during the green traffic-signal duration, and fully blocked during the remaining time.

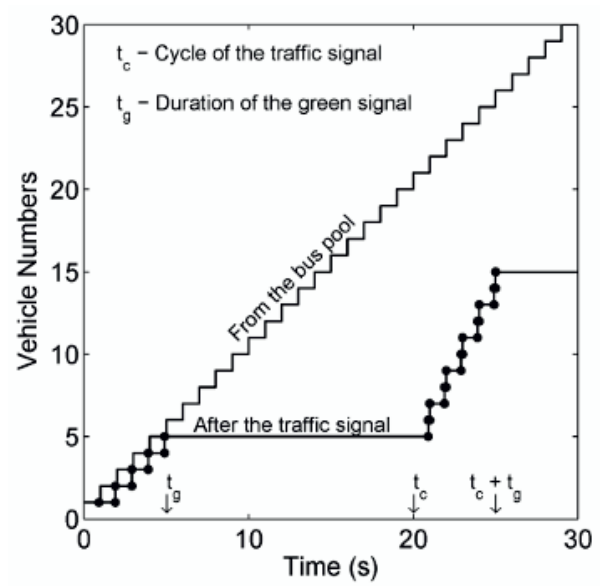

Figure 8. A test case of the traffic signal sub-system

\section{Numerical Trials: Simple Corridor}

We established a small-symmetric BRT network for this case of numerical trials. The network consists of a bus pool and two BRT stations that are connected by two road-segments. In the simulation, two buses were dispatched from the bus pool and entered the network. Once the buses were in the network, they would circulate from Station 1 to Station 2, then, from Station 2 to Station 1, and so on. The network is shown in Fig. 9 as a SimEvents model.

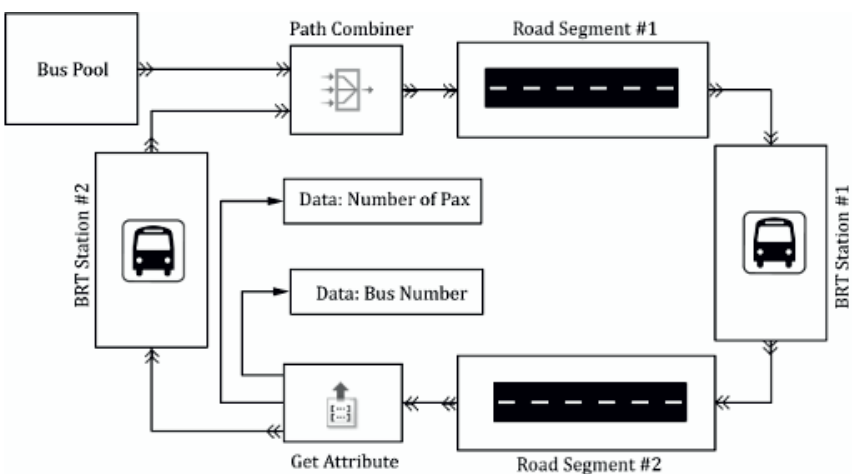

Figure 9. The SimEvents-numerical model of the two-station corridor.

Besides the sub-systems of the road-segments, the bus pool and BRT stations, the SimEvents model above also has other sub-systems: Path Combiner and Get Attribute. The earlier sub-system allows the bus, either from the bus pool or from the station \#2, to travel to the road segment \#1. The latter sub-system allows us to extract the bus internal data or the bus attribute data; in this case, the data were the bus number and the number of passengers on board.

A complete list of the model parameters, associated with the SimEvents-numerical model, is given in Table 2.

Table 2. Parameters of the simple corridor model.

\begin{tabular}{|c|c|c|}
\hline Model Parameters & Characteristics & Value \\
\hline BRT Station: & & \\
\hline $\begin{array}{l}\text { Passenger inter-arrival } \\
\text { time }\end{array}$ & Exp. Dist. & $\begin{array}{l}\text { Mean }=1 \\
\text { person } / \min \end{array}$ \\
\hline Number of alighted pax & Uniform Dist. & $\begin{array}{l}\text { Min. }=0, \\
\text { Max. }=25 \\
\text { persons }\end{array}$ \\
\hline Bus service time & Triangle Dist. & $\begin{array}{l}\text { Min. }=1 \mathrm{~min}, \\
\text { Max. }=3 \mathrm{~min} \text {, } \\
\text { Mode }=2 \mathrm{~min}\end{array}$ \\
\hline $\begin{array}{l}\text { Road Segment: } \\
\text { Travel time }\end{array}$ & Exp. Dist. & Mean $=5 \mathrm{~min}$ \\
\hline $\begin{array}{l}\text { Bus Pool: } \\
\text { Bus inter-departure time }\end{array}$ & Deterministic & $5 \mathrm{~min}$ \\
\hline
\end{tabular}

Those parameters were for the BRT stations, the roadsegments, and the bus pool. The two BRT station were assumed to be identical, and so do the two roadsegments. As for the BRT station, the passengers were assumed to arrive according to a Poisson process with an arrival rate of 1 person $/ \mathrm{min}$, slightly lower than that observed by ref. [25]. The number of alighted passengers was assumed to be discretely and uniformly distributed between 0 and 25 persons. Meanwhile, the bus service time was short with a mode of $2 \mathrm{~min}$. As for the roadsegment, the bus was assumed to travel with a duration following the exponential distribution function with a mean of $5 \mathrm{~min}$. The exponential distribution for the bus traveling time was also observed by ref. [26]. Finally, the bus inter-departure time was set to exactly be $5 \mathrm{~min}$ that was based on the BRT standard for the bus frequency during the rush-hours [3].

The time frame for the simulation was 24 hours although BRT usually operates about 18 hours per day, and the analysis was replicated for 200 days. The simulation results are reproduced in Figs. 10-12. 


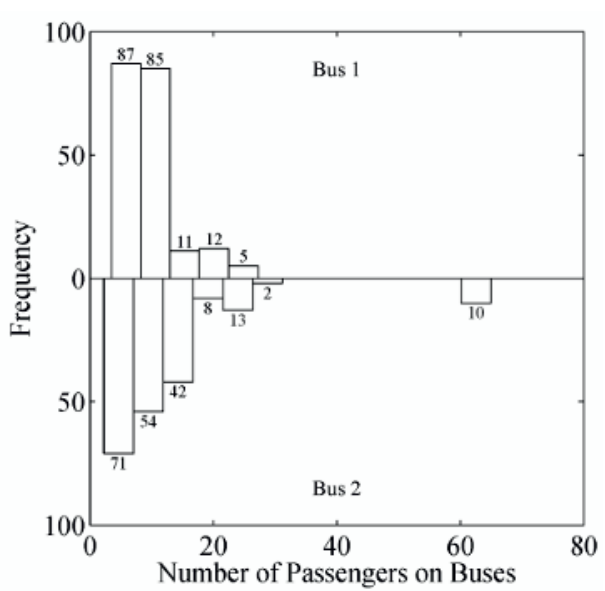

Figure 10. Bi-histogram of the distribution of the time-averaged number of passengers on both the buses in steady-state condition for 200 replications.

The results in Fig. 10 shows the distribution of the number of passengers on the time-averaged sense on both the buses when the analysis has reached its steady-state condition. The figure reveals an interesting phenomenon that the distribution of the time-averaged number of passengers was rather similar for both buses. This fact seems acceptable by considering the model design and its input parameters that were designed to be symmetric. The actual numbers of passengers on the two buses were 14 passengers on average with 18 passengers' deviation. When the buses left the BRT Station \#2, they were boarded with about 8 time-averaged numbers of the passengers for $85 \%$ of the cases. On a few cases, the second bus was boarded by about 60 passengers. Variation of the number of passengers was much wider on the second bus, about twice wider than that on the first bus.

The time history data of the number of passengers are reproduced in Fig. 11 for the first bus and in Fig. 12 for the second bus. On the right side of each figure, a boxplot is provided to show the final state of the time-averaged number of passengers for 200 replications. In general, the number of passengers was highly fluctuating in the range of 0 to about 70 passengers. For the historical data depicted in the figure, the passengers were on board for $62 \%$ of the time for the bus 1 and $55 \%$ of the time for the bus 2 .

\section{Conclusions}

This paper has discussed a potential use of the simulation framework to model the dynamics of a bus-rapid transit system. The framework was developed on the basis of the standard features existed in a common discrete-event simulation system. For this particular application, significant complexity of modeling was found in modeling a BRT station. A few assumptions have to be made to allow development of the sub-system. The established model, in limited numerical trials, systematically produced well observed phenomena of the actual BRT system.

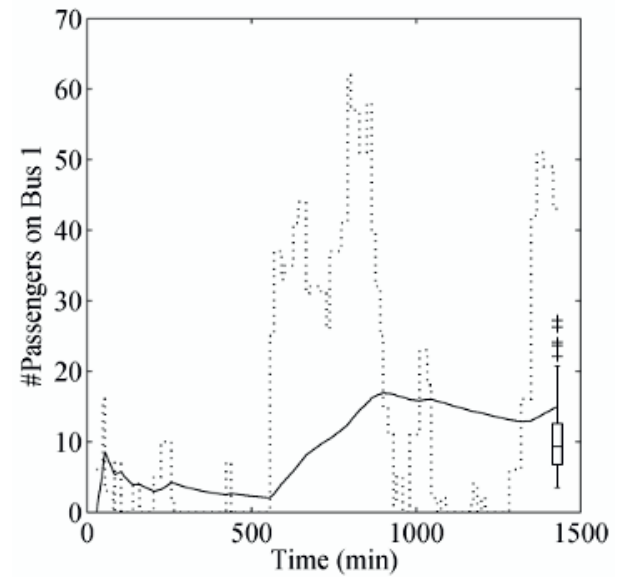

Figure 11. The history of the number of passengers on Bus 1 (broken line), time-averaged (solid line), and a boxplot of the time-averaged number of passengers at the end of the analysis.

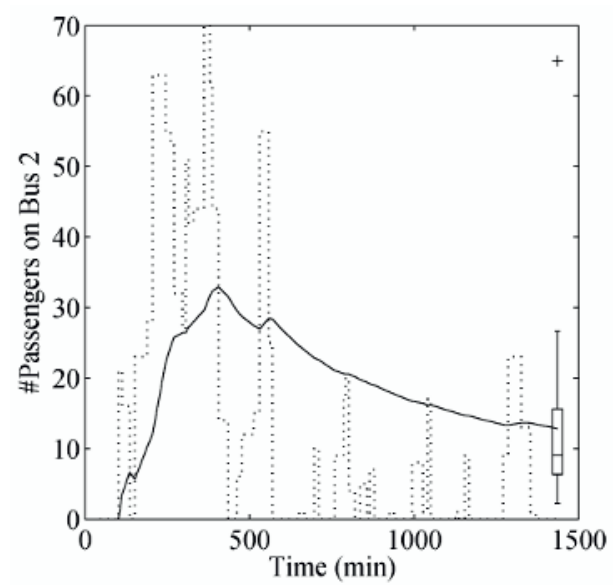

Figure 12. The history of the number of passengers on Bus 2 (broken line), time-averaged (solid line), and a boxplot of the time-averaged number of passengers at the end of the analysis.

\section{References}

1. S. Morichi, Journal of the Eastern Asia Society for Transportation Studies 6, 1 (2005)

2. C. Campo, Master's thesis, School of civil and environmental engineering, Georgia Institute of Technology (2010), http://hdl.handle.net/1863/37089

3. A. Weinstock, W. Hook, M. Replogle, R. Cruz, Tech. rep., Institute for Transportation and Development Policy (2011)

4. L.V. Bin, N. Huimin, Journal of transportation system engineering and information technology 11, 45 (2011)

5. J.M. Lehtonen, U. Seppala, Integrated manufacturing systems 8, 351 (1997)

6. S. Gao, Z. Wu, Journal of transportation systems engineering and information technology 11, 124 (2011)

7. A. Visser, A.J. van der Wees, L.O. Hertzberger, Discrete event modelling methodology for intelligent transport systems, in Proceedings of the world congress on intelligent transport systems (Torino, Italy, 2000), p. 2016 
8. G. Alves, J. Rosmann, R. Wischnewski, World academy of science, engineering, and technology 58, 821 (2009)

9. R.C. Dos Santos Silve, T.B. Brito, R.C. Botter, N.N. Pereira, Modeling of a closed-loop maritime transportation system with discrete event simulation and multi-criteria decision analysis, in proceedings of the world congress on engineering and compuer science (San Francisso, USA, 2011), Vol. II

10. K. Valiguran, M. Folting, M. Blaho, Transport system realization in simevents tool, http://dsp.vscht.cz/konference matlab/MATLAB09/ prispevky/107_valigura.pdf (2012)

11. V. Ancora, C. Nelli, M. Petrelli, Proceedia Social and Behavioral Sciences 54, 1250 (2012)

12. L. Yu, L. Yu, X. Chen, T. Wan, J. Guo, Journal of Public Transportation 9, 239 (2006)

13. H. Li, B. Mao, R.L. Bertini, Evaluating the impacts of bus facility design features on transit operations in Beijing, China: A simulation approach, in $87^{\text {th }}$ annual meeting of the transportation research board (2008)

14. R. Widanapathiranage, J.M. Bunker, A. Bhaskar, A microscopic simulation model to estimate bus rapid transit (BRT) station service capacity with mixed stopping and non-stopping bus operation, in OPTIMUM 2013: International symposium on recent advances in transport modelling (2013)

15. Fergyanto E. Gunawan, E. Kusnandar, Jurnal jalan dan jembatan 28, (2011), http://digilib.polban.ac.id/gdl.php?mod=browse\&opread\&id=jbptpolban-gdl-fergyantoe-3251

16. B. Seibold, M. R. Flynn, A. R. Kasimov, R. R. Rosales, Constructing set-valued fundamental diagrams from jamiton solutions in second order traffic models (2013), http://arxiv.org/pdf/1204.5510v2

17. M.R. Flynn, A.R. Kasimov, J.C. Nave, R.R. Rosales, B. Seibold, On “Jamitons", self-sustained nonlinear traffic waves, Online Article (2008), http://arxiv.org/abs/0809.2828

18. M.H. Kabir, Master's thesis, Department of Mathematics, Jahangirnagar University, Bangladesh (2008)

19. TransJakarta profile book (2012)

20. C.A. O'Flaherty, D.O. Mangan, Traffic engineering and control 11, 419 (1970)

21. M.D. Salek, R.B. Machemehl, Tec. Rep. Research Report 167211-1, Center for Transportation Research (1999), http://swutc.tamu.edu/publications/technicalreports/1 67211-1.pdf

22. W. Fan, R. B. Machemehl, Characterizing bus transit passenger waiting times, in 2nd material specialty conference of the Canadian society for civil engineering (Montreal, Quebec, Canada, 2002)

23. M. Luethi, U. Weidmann, A. Nash, Passenger arrival rates at public transport stations, Institute for transport planning and systems, ETH Zurich (2006), retrieved on July 2012, http://www.andynash.net/nashpublications/Luethi2007-pax-arrivals-TRB-paper.pdf
24. M. K. Islam, U. Vandebona, Reliability analysis of public transit systems using stochastic simulation, in 33rd Australasian transport research forum conference (Canberra, Australia, 2010), http://www.worldtransitresearch.info/cgi/viewconten t.cgi? article $=5072 \&$ context $=$ research

25. Fergyanto E. Gunawan, E. Kusnandar, B. S. Abbas, A. Mazaya, C. Rijal, I.A. Permatasari, Journal of transportation system engineering and information technology (2013) (In review process)

26. Fergyanto E. Gunawan, Erwin Kusnandar, Bahtiar Saleh Abbas, Ferdy L Pranolo, Tehdy Hartono, and Victor Pangestu. Travel time reliability of transjakarta, Indonesia bus rapid transit. Journal of Transportation Systems Engineering and Information Technology (2013) (In review process)

27. E E Osuna and G F Newell. Transportation Science, 21, 55 (1972)

28. E M Holroyd and D A Scraggs. Traffic Engineering and Control, 8, 158 (1966)

29. P I Welding. Operational research quarterly, 8, 133 (1957)

30. P A Seddon and M P Day. Traffic engineering and control, 15, 422 (1974)

31. Fergyanto E. Gunawan. International journal of transportation systems and information technology 12, 77 (2012)

32. Martin Treiber, A Martin Treiber, Ansgar Hennecke, and Dirk Helbing, Physical Review E, 62, 1805, (2000) http://link.aps.org/doi/10.1103/PhysRevE.62.1805 